\title{
NEAR PERFECT RECONSTRUCTION FILTER BANKS FOR POWER QUALITY ANALYSIS
}

\section{Dimitar Taskovski, Ljubica Koleva, Aleksandar Milchevski, Vladimir Dimcev}

Faculty of Electrical Engineering and Information Technologies, Ss Cyril and Methodius University - Skopje, 1000 Skopje, R. Macedonia (e-mail:dtaskov@feit.ukim.edu.mk).

\begin{abstract}
The wavelet transform has been successfully used in the area of power quality analysis. There are many published papers with methods for power quality disturbance classification or harmonics measurement, which use wavelet transform. However, the properties of the wavelet transform can drastically vary from the choice of the wavelet. In this paper we analyze the influence of the choice of the wavelet to the accuracy of the power quality classification method and to high frequency harmonics measurements. Additionally to the well known wavelet filters we introduce near perfect reconstruction filter banks. The simulation results indicate that these filter banks are a good choice for classification of power quality disturbances, especially in the presence of noise and for high frequency harmonics measurements.
\end{abstract}

Keywords: Power quality, power harmonics, disturbance classification, quadrature mirror filters.

(C) 2013 Polish Academy of Sciences. All rights reserved

\section{Introduction}

The use of modern electronic equipment leads to disturbances in the power network quality. In [1], the authors have analyzed that the poor power quality accounts for losses adding up to $€ 150$ billion, annually, only in the European Union. The main cause of the losses is said to be power interruptions such as dips, surges, transients and short interrupts. The effects on the equipment from the power quality (PQ) disturbances can be found in the IEEE Std. 1159-2009, [2], e.g. transient overvoltages can result in immediate dielectric failure in all classes of equipment.

Solving power quality problems first of all means detecting the disturbance sources and then eliminating them. It is very important that the detection can be performed automatically. So, the classification of the disturbances in the power system signals is a very important issue. Several systems for automatic detection and classification of PQ disturbances have been proposed. Rule-based expert systems, fuzzy classification systems, artificial neural networks (ANN) and support vector machines (SVM) are the most common classifiers based on artificial intelligence [3]. To classify the power quality events, feature vectors obtained from the waveforms are used. Different signal processing techniques are used for feature extraction in PQ events [4], speaking of which, the discrete wavelet transform (DWT) notes extensive use in the past years [5]. Main advantage of using DWT in power quality analysis is its ability to analyze the signal simultaneously in the time and frequency domains. This makes the transform highly suitable for analysis of non-stationary signals. Since the energy of the wavelet coefficients is varying over different scales depending on the input signal, the energy of the subbands could be used for construction of a feature vector for training and testing.

Additionally, many wavelet-based algorithms for the harmonic analysis in power systems are proposed [6]-[8]. The presented results are competitive with the results obtained using 
discrete Fourier transform (DFT), as proposed in the IEC standard 61000-4-7 [9].

The DWT and inverse DWT can be implemented using a multistage two-channel filter bank with the scaling and wavelet function associated with its low-pass filter and its high-pass filter, respectively. It is well known that improperly designed analysis/synthesis filter banks can cause aliasing, amplitude distortion and phase distortion. The design of a two-channel filter bank with perfect reconstruction (PR) property is a well-known issue [10]. Large numbers of filter banks (wavelet families) that satisfy the PR property are proposed in the literature, Daubechies, Coiflets and Symlets among them. Also, all these wavelet families are mainly used in power quality applications, and Vaidyanathan with 24 coefficients (v24), Daubechies with 20 coefficients (db20) and Coiflet 5 with 30 coefficients (coif5) are proposed in the literature as the most adequate for harmonic analysis, and the fourth-order Daubechies filter (db4) is proposed as most appropriate for power quality disturbance classification.

However, for PQ-related applications (harmonics measurement, detection and classification of power quality disturbances), wavelets are used for analyzing the voltage/current waveform only. There is no need of signal synthesis. It is doubtful whether keeping the PR property still makes sense in this case. Relaxing this condition will allow more freedom for the optimization, and a well-designed near-perfect reconstruction (NPR) filter bank could outperform a PR filter bank because of better performance in, for example, stopband attenuation or computational complexity.

In [11], the authors proposed the use of NPR quadrature mirror filter (QMF) banks as possible solution for harmonics measurement. Compared to well-known wavelet families, the introduced Johnston filters [12] have better selectivity properties which make them suitable for harmonic analysis. The presented results show that Johnston filters outperform the commonly-used wavelet filters for harmonic analysis, especially in case of non-stationary signals.

This paper is an extended version of the paper [13] where the influence of NPR QMF to the overall accuracy of the wavelet-based classification method is analyzed. Additionally, the properties of NPR QMF filter banks are examined in case of higher frequency harmonics measurement.

\section{Wavelet transform}

\subsection{Discrete wavelet transform}

The discrete wavelet transform (DWT) is given with:

$$
D W T[m, n]=\frac{1}{\sqrt{\tau_{0}^{m}}} \sum_{k=-\infty}^{\infty} f[k] \psi *\left[\frac{k-n c_{0} \tau_{0}^{m}}{\tau_{0}^{m}}\right],
$$

where function $\psi$ is the base function or the mother wavelet, and $c$ and $\tau$, are the dilation and translation parameters, respectively. With the choice $c_{0}=2$ and $\tau_{0}=1$, a dyadic orthonormal wavelet transform is obtained [14]. In this case DWT can be easily and quickly implemented by filter bank techniques. The filter bank is used to decompose the signal into various levels using a low-pass filter with a transfer function $H_{0}(z)$ and a high-pass filter with a transfer function $H_{1}(z)$, as shown in Fig. 1(b). The obtained low-pass coefficients represent a coarse approximation of the input signal and high-pass coefficients represent the "added detail" signal. The low frequency part (approximation signal) is split again into two parts of high and low frequencies. Depending on the application at hand and on the size of the input signal, the process could be repeated several times. As a result, logarithmic decomposition of the 
frequency spectra of the input signal is obtained. With the inverse DWT coefficients, the original signal can be reconstructed from the approximation signal and details.
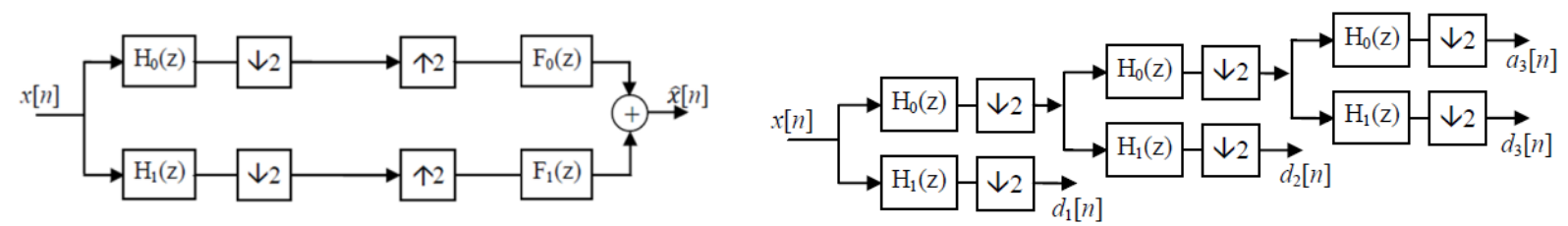

Fig. 1. (a) Two-band filter bank, (b) Wavelet decomposition over 3 levels.

\subsection{Wavelet packet transform}

The wavelet packet transform (WPT) is a generalization of DWT. The difference is that in the WPT signal decomposition, both the approximation and detail coefficients are further decomposed at each level and as the result a uniform frequency decomposition of the input signal is obtained. The number of output bands for $L$-level decomposition is $2^{L}$. With the selection of an adequate sampling frequency and the level of the decomposition these uniform frequency bands can be used for harmonics measurement of the input signal [6].

\section{Quadrature mirror filter banks}

The basic structure of a two-band filter bank is shown in Fig. 1(a). $H_{0}(z)$ and $H_{1}(z)$ designate the low-pass and high-pass analysis filters, respectively, and $F_{0}(z)$ and $F_{1}(z)$ designate the low-pass and high-pass synthesis filters, respectively. With $\downarrow 2$ and $\uparrow 2$ the downsampling and the upsampling operator are denoted. The downsampler reduces the size of the input signal by omitting every other sample and the upsampler increases the size of the input signal by inserting a zero between the values of the input signal. The input-output relation of a two-band filter bank is given by:

$$
\hat{X}(z)=\frac{1}{2}\left[H_{0}(z) F_{0}(z)+H_{1}(z) F_{1}(z)\right] X(z)+\frac{1}{2}\left[H_{0}(-z) F_{0}(z)+H_{1}(-z) F_{1}(z)\right] X(-z) .
$$

The first term describes the transmission of the signal through the system, while the second term represents an aliasing error due to the change of the sampling rate in the filter bank. The simplest way to cancel the aliasing is by selecting the filters in the analysis stage as $H_{1}(z)=H_{0}(-z)$ and by selecting the synthesis filter as $F_{0}(z)=H_{0}(z), F_{1}(z)=-H_{1}(z)$. Since the mirror-image symmetry about the frequency $(\omega=\pi / 2)$ exists between $H_{1}(z)=H_{0}(-z)$, these filters are known as quadrature mirror filters (QMF) [10]. With filters chosen as above, the last equation becomes:

$$
\hat{X}(z)=\frac{1}{2}\left[H_{0}^{2}(z)-H_{1}^{2}(z)\right] X(z)=\frac{1}{2}\left[H_{0}^{2}(z)-H_{0}^{2}(-z)\right] X(z)=H(z) X(z) .
$$

In this way, the whole filter bank is completely defined by a single low-pass filter $H_{0}(z)$. Additionally, if $H_{0}(z)$ is FIR and has a linear phase, then the overall transfer function $H(z)$ will have a linear phase, so phase distortion is eliminated. This means that $h_{0}[n]$ is symmetric. That is, $h_{0}[n]=h_{0}[N-n]$ for a filter of order $N$. Then,

$$
H_{0}\left(e^{j \omega}\right)=e^{-j \omega N / 2} R(\omega),
$$

where $R(\omega)$ is real for all $\omega$. From this and the observation that $\left|H_{0}\left(\mathrm{e}^{\mathrm{j} \omega}\right)\right|$ is even [10], 


$$
H\left(e^{j \omega}\right)=\frac{e^{-j \omega N / 2}}{2}\left(\left|H_{0}\left(e^{j \omega}\right)\right|^{2}-(-1)^{N}\left|H_{0}\left(e^{j(\pi-\omega)}\right)\right|^{2}\right)
$$

In order to avoid severe distortion around $\omega=\pi, N$ must be even. Therefore,

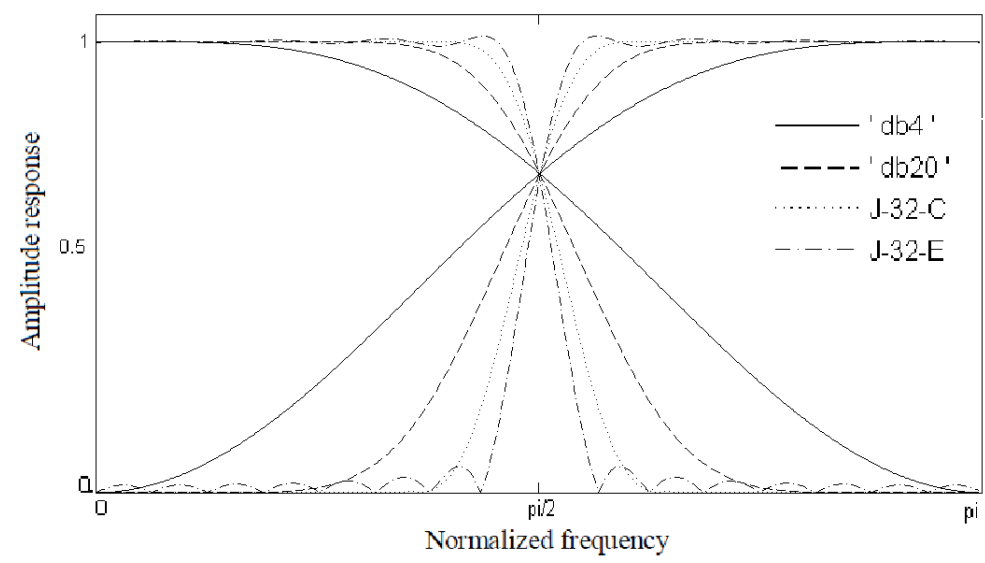

Fig. 2. Frequency responses of different filters: Johnston filters with 32 coefficients and different transition bands (32C and 32E), Daubechies with 4 coefficients (db4) and Daubechies with 20 coefficients (db20).

$$
H\left(e^{j \omega}\right)=\frac{e^{-j \omega N / 2}}{2}\left(\left|H_{0}\left(e^{j \omega}\right)\right|^{2}+\left|H_{0}\left(e^{j(\pi-\omega)}\right)\right|^{2}\right) .
$$

Since this transfer function determines the amplitude distortion present in the signal, it would be desirable to find filter coefficients such that

$$
\left|H_{0}\left(e^{j \omega}\right)\right|^{2}+\left|H_{1}\left(e^{j \omega}\right)\right|^{2} \approx 1 .
$$

From the above, it is clear that the design of a quadrature mirror filter is essentially a problem of finding a low-pass filter $H_{0}(z)$ with good stopband attenuation at the same time maintaining $\left|H\left(e^{j \omega}\right)\right| \approx 1$ for all $\omega$, where $H\left(e^{j \omega}\right)$ is the frequency response of the overall system.

Johnston [12] has devised a technique to minimize amplitude distortion based on wellknown multivariable optimization routines. Essentially, the filter coefficients are iteratively adjusted in order to minimize the value of an objective function that represents a linear combination of the overall passband error $E_{r}$ and the stopband energy of the low-pass analysis filter of the filter bank $E_{s}$ :

$$
E=E_{r}+\alpha E_{s}
$$

where

$$
E_{r}=\int_{\omega=0}^{\pi}\left[\left|H_{0}\left(e^{j \omega}\right)\right|^{2}+\left|H_{0}\left(e^{j(\omega+\pi)}\right)\right|^{2}-1\right]^{2} d \omega
$$

and

$$
E_{s}=\int_{\omega=\omega_{s}}^{\pi}\left|H_{0}\left(e^{j \omega}\right)\right|^{2} d \omega .
$$

The parameter $\alpha$ in (8) is a positive weight that can be used to control stopband attenuation for filter $H_{0}$, and $\omega_{s}$ is the frequency of the stopband edge. 
Fig. 2 shows the frequency response of four different filters used in the filter banks. Frequency responses of Johnston $\mathrm{J} 32 \mathrm{C}$ and $\mathrm{J} 32 \mathrm{E}$ filters show a reduction of overlapping in the transition band compared to Daubechies filters with length 4 and 20.

One important property of the QMF banks is their efficient implementation due to the modulated structure, where the high-pass and low-pass filters are related as $H_{1}(z)=H_{0}(-z)$. Their polyphase components are given with [10]:

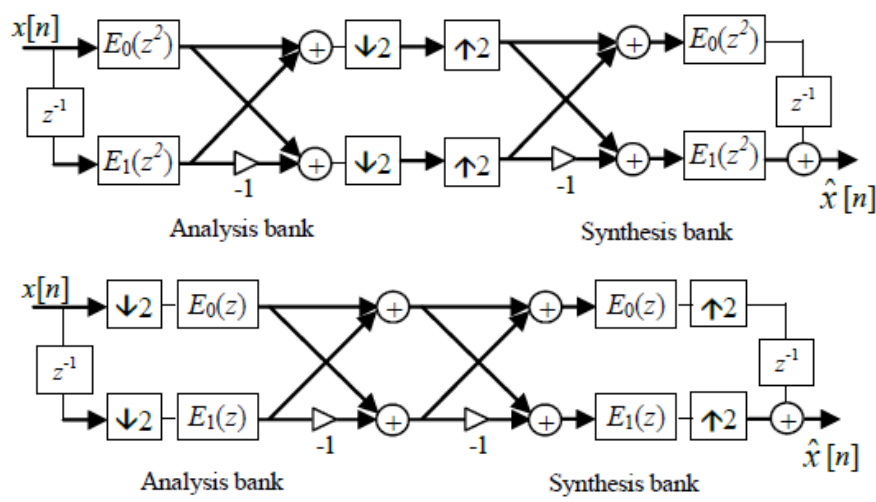

Fig. 3. (a) Polyphase implementation of QMF bank, (b) Efficient implementation using noble identities.

$$
\begin{aligned}
& H_{0}(z)=E_{0}\left(z^{2}\right)+z^{-1} E_{1}\left(z^{2}\right) \\
& H_{1}(z)=E_{0}\left(z^{2}\right)-z^{-1} E_{1}\left(z^{2}\right)
\end{aligned}
$$

With the last two equations, the analysis filter bank can be represented as in Fig. 3 (a), and by using the noble identities [10] as in Fig. 3 (b). With these modifications same structures are used for implementation of both filters and the sampling rate is reduced by two. As a result, the number of operations is reduced four times in comparison with a standard filter bank implementation.

\section{Classification}

The support vector is chosen as a machine learning method. We provide a brief description of the SVM method and descriptions for the feature extraction process.

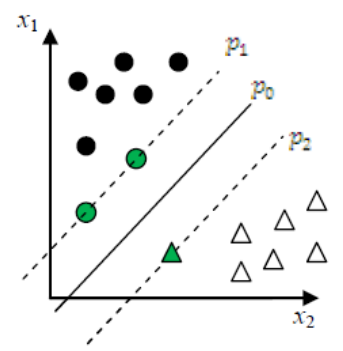

Fig. 4. Classification of two classes.

\subsection{Support Vector Machine}

Support Vector Machines (SVM) is one of the most popular machine learning algorithms. Many experts believe it is the best learning algorithm, because of the vast number of successful applications and the solid theoretical foundation. Another reason for using SVM is the ease of use and the intuitive interpretation of the algorithm. Fig. 4. illustrates a binary classification problem. The samples from the first class are depicted as circles and the 
samples from the second class are depicted as triangles. The classification problem is solved by finding the hyper-plane $p_{0}$ defined as:

$$
p_{0}: w \cdot x-b=0 .
$$

The hyper-plane is obtained as a maximum margin hyper-plane, by using a set of training examples with known class labels. However, by finding this hyper-plane a prediction can be made for the class label of a new example which was not included in the training set. Let the training examples be denoted with $x_{i}$ and the class labels are -1 and 1, i.e. $c_{i}=1$ if the training example belongs to the first class and $c_{i}=-1$ if the training example belongs to the second class. The classification problem can be restated as finding two hyper-planes which separate the data from the two classes and are as far as possible from each other. The two hyperplanes can be defined as:

$$
\begin{aligned}
& p_{1}: w \cdot x-b=1 \\
& p_{2}: w \cdot x-b=-1
\end{aligned} .
$$

The margin, i.e. the distance between the two hyper-planes is $\frac{2}{\|w\|}$. The classification problem can be solved by maximizing the margin. However, it is easier to solve the following problem, which also leads to the same solution, yet the optimization function is quadratic:

$$
\begin{aligned}
& \text { Minimize } \frac{1}{2}\|w\|^{2} \\
& \text { subject to (for any } i=1, . ., n) \\
& c_{i}\left(w \cdot x_{i}-b\right) \geq 1
\end{aligned}
$$

Introducing Lagrange multipliers, $\alpha_{i}$ the optimization problem is transformed into:

$$
\min _{w, b} \max _{\alpha_{i}}\left\{\frac{1}{2}\|w\|^{2}-\sum_{i=1}^{n} \alpha_{i}\left[c_{i}\left(w \cdot x_{i}-b\right)-1\right]\right\} .
$$

Most of the Lagrange multipliers, $\alpha_{i}$, will be set to zero, as a solution of the optimization problem, because the corresponding term $\left[c_{i}\left(w \cdot x_{i}-b\right)-1\right]$ will be positive. The $\alpha_{i}$ which are not set to zero correspond to $x_{i}$, called support vectors.

In [15] and [16] a soft margin SVM was introduced, in order to allow mislabeled examples. Also if the classes are not linearly separable a kernel trick is used, i.e. the dot product is replaced by a nonlinear kernel function.

For the needs of classification, in this work we have utilized a linear multiclass SVM with the use of the implementation of libSVM [17].

\subsection{Feature extraction}

For the feature extraction process we utilize the DWT. The signal, which is tested for PQ disturbances, is first decomposed in l-levels using DWT. The energy of the detail and approximation coefficients at each level of decomposition is used as a feature vector, and is calculated according to the following equations:

$$
E D_{m}=\sum_{n=1}^{N} d_{m, n}^{2}, \quad m=1, \ldots, l,
$$




$$
E A_{l}=\sum_{n=1}^{N} a_{l, n}^{2}
$$

where $d_{m, n}, m=1, \ldots, l$ is the wavelet detail coefficient in the wavelet decomposition from level 1 to level $l$ and $a_{l, n}$ is the wavelet approximation coefficient in the wavelet decomposition at level $l . N$ is the total number of wavelet coefficients at each level of decomposition, $E D_{m}$ is the energy of detail coefficients at the decomposition level $m$ and $E A_{l}$ is the energy of the approximate wavelet coefficients at decomposition level $l$. In this way, the size of the analyzed data is significantly reduced and the original waveform is represented with only $l+1$ coefficients. We have chosen $l=7$ as the optimal number of levels of decomposition. Further increment of the level does no longer increase the accuracy of the algorithm and a too large number of levels of decomposition can even decrease the accuracy of the algorithm.

\section{Harmonics measurement}

As mentioned above the WPT decomposes the signal into uniform frequency bands. By adequately selecting the sampling frequency and the decomposition tree, the obtained frequency bands can be used for measurement of the different harmonic components of the input signal. For instance, with the sampling frequency set to $1600 \mathrm{~Hz}$ and with three levels of decomposition, eight frequency bands with $100 \mathrm{~Hz}$ interval are obtained and can be used for measurement of odd harmonics up to the 15-th harmonic. The rms of the signal can be computed directly from the wavelet coefficients in the last bands [7].

The introduction of equipment with electronic components will involve high-frequency distortions with frequencies of several $\mathrm{kHz}$ [18]. In order to analyze these high frequency disturbances with wavelet transform, a higher sampling frequency is required. The increase of the sampling frequency implies the use of a deeper wavelet decomposition tree in order to obtain the same width of the output frequency bands. All this will have an influence on the frequency characteristics of the output bands at the last level of decomposition, and consequently on the obtained results. This influence is illustrated in Fig. 5, where the frequency responses of each output band obtained after three levels of decomposition using a Johnston 64E filter and Vaidyanathan v24 are given. The frequency response of each output band is different and depends on the type and the sequence of filters (LP or HP) through which the input signal proceeds to the final band. The spectral leakage which is especially evident in the central frequency bands can be observed. Obviously, the use of filters with better selectivity properties will lead to more accurate harmonics measurement.

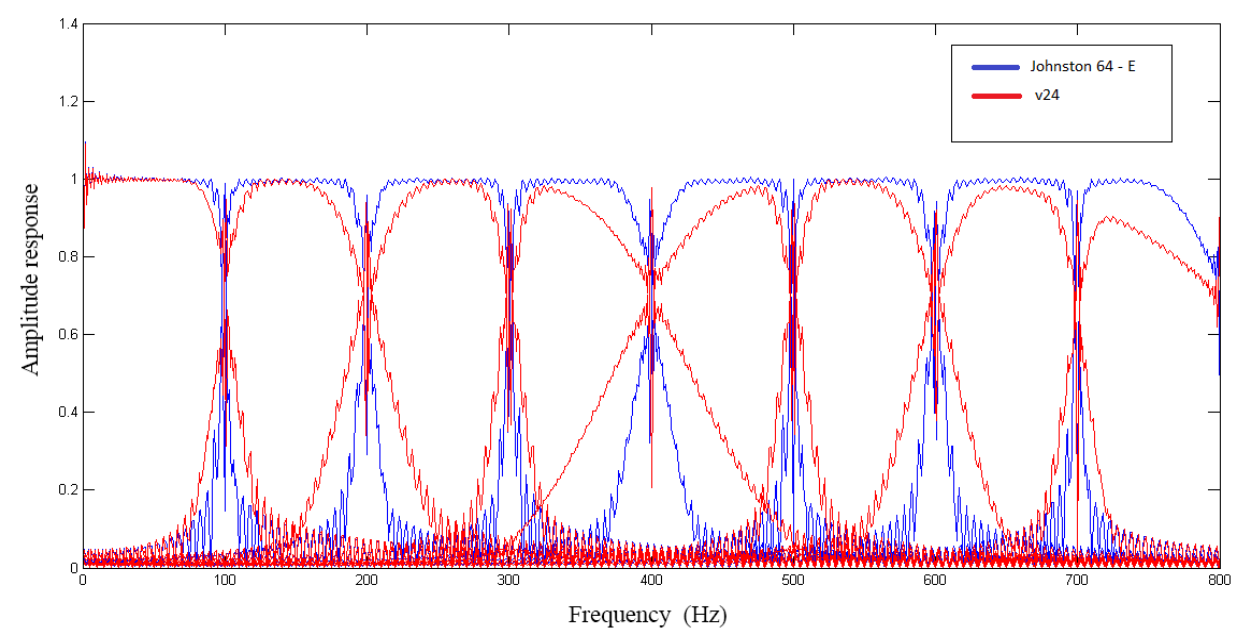

Fig. 5. Frequency characteristics of Johnston 64E filter and v24 after three level of decomposition. 
Table 1. Comparison between rms values using different filters in case of existence of higher-order harmonics

\begin{tabular}{|c|c|c|c|c|c|c|}
\hline $\begin{array}{c}\text { Harmonic } \\
\text { order }\end{array}$ & $\begin{array}{c}\text { Magnitude } \\
\text { in \% }\end{array}$ & db20 & db40 & v24 & J32D & J64E \\
\hline $1^{\text {st }}$ & $\mathbf{1 0 0}$ & 99,95 & 100.4 & 100.0 & 100.0 & 100.0 \\
\hline $3^{\text {rd }}$ & $\mathbf{3 . 4 5}$ & 4.620 & 3.306 & 3.323 & 3.255 & 3.481 \\
\hline $5^{\text {th }}$ & $\mathbf{1 . 7 1}$ & 1.479 & 1.977 & 1.949 & 1.738 & 1.713 \\
\hline $7^{\text {th }}$ & $\mathbf{1 . 1 5 5}$ & 1.300 & 1.230 & 0.956 & 1.225 & 1.136 \\
\hline $9^{\text {th }}$ & $\mathbf{0 . 6}$ & 0.095 & 0.348 & 0.893 & 0.555 & 0.603 \\
\hline $11^{\text {th }}$ & $\mathbf{0 . 4 9 5}$ & 0.464 & 0.488 & 0.511 & 0.490 & 0.496 \\
\hline $13^{\text {th }}$ & $\mathbf{0 . 3 1 5}$ & 0.397 & 0.361 & 0.291 & 0.315 & 0.315 \\
\hline $15^{\text {th }}$ & $\mathbf{0 . 2 2 5}$ & 0.273 & 0.171 & 0.296 & 0.149 & 0.212 \\
\hline $17^{\text {th }}$ & $\mathbf{0 . 1 9 8}$ & 0.127 & 0.248 & 0.087 & 0.254 & 0.220 \\
\hline $19^{\text {th }}$ & $\mathbf{0 . 1 7 7}$ & 0.086 & 0.170 & 0.144 & 0.177 & 0.178 \\
\hline $21^{\text {st }}$ & $\mathbf{0 . 1 6}$ & 0.117 & 0.143 & 0.174 & 0.164 & 0.160 \\
\hline $23^{\text {rd }}$ & $\mathbf{0 . 1 4 7}$ & 0.160 & 0.184 & 0.167 & 0.160 & 0.146 \\
\hline $25^{\text {th }}$ & $\mathbf{0 . 1 3 5}$ & 0.083 & 0.094 & 0.115 & 0.121 & 0.136 \\
\hline $27^{\text {th }}$ & $\mathbf{0 . 1 2 5}$ & 0.073 & 0.153 & 0.145 & 0.119 & 0.126 \\
\hline $29^{\text {th }}$ & $\mathbf{0 . 1 1 6}$ & 0.063 & 0.126 & 0.137 & 0.128 & 0.116 \\
\hline $31^{\text {st }}$ & $\mathbf{0 . 1 0 9}$ & 0.024 & 0.132 & 0.148 & 0.401 & 0.121 \\
\hline $33^{\text {rd }}$ & $\mathbf{0 . 1 0 2}$ & 0.146 & 0.017 & 0.025 & 0.104 & 0.103 \\
\hline $35^{\text {th }}$ & $\mathbf{0 . 0 9 6}$ & 0.157 & 0.058 & 0.050 & 0.097 & 0.096 \\
\hline $37^{\text {th }}$ & $\mathbf{0 . 0 9 1}$ & 0.109 & 0.066 & 0.064 & 0.092 & 0.091 \\
\hline $39^{\text {th }}$ & $\mathbf{0 . 0 8 6}$ & 0.103 & 0.068 & 0.076 & 0.085 & 0.086 \\
\hline
\end{tabular}

\section{Experimental results}

\subsection{Choice of the filters}

As already mentioned, the properties of the DWT and WPT significantly depend on the choice of the wavelet filters. Different wavelets decompose the analyzed signal in a different way, thus affecting the overall accuracy of the classification method or the process of harmonics measurement. The Johnston filters [12] with 24, 32 and 64 coefficients (J24B, $\mathrm{J} 24 \mathrm{C}, \mathrm{J} 24 \mathrm{D}, \mathrm{J} 32 \mathrm{C}, \mathrm{J} 32 \mathrm{D}, \mathrm{J} 32 \mathrm{E}, \mathrm{J} 64 \mathrm{E}$ ) are used in this work, besides well-known wavelet families. Letter $\mathrm{C}$ is a notation for all Johnston filters that have a normalized transition band of 0.0625 , D refers to a normalized transition band of 0.043 and E refers to 0.023 .

\subsection{Harmonics measurement}

In order to investigate the influence of different wavelet filters on the high frequency harmonic analysis of power system waveforms, we have implemented the wavelet packet transform-based algorithm given in [7]. In this work we used $50 \mathrm{~Hz}$ input waveforms sampled at $6400 \mathrm{~Hz}$. After applying five level WPT decomposition, the obtained 32 frequency bands are in the range of $100 \mathrm{~Hz}$ and can be used for measurement of odd harmonics up to the 64th harmonic.

The test signals that are used to examine the filters' influence are generated according to the limitations for harmonics maximum magnitude (class B), given in IEC 61000-3-2 Standard [19]. Table 1 shows the results of the rms values using different filters in case of existence of higher order harmonics. All of the rms values are calculated after performing five levels of decomposition. It can be noticed that commonly used wavelet filters with number of coefficients between 20 and 32 cannot offer satisfactory reliability. Some authors propose the use of higher-order filters to avoid leakage and divergence from the true values. In [20], Daubechies filter db40 is proposed for harmonic analysis. We have included it in our experiments, but there was no significant improvement in the computed rms values. The lack of good performance of these filters is a result of the increased number of decompositions, 
thus the frequency characteristics of the output bands are subject to larger modification. It can be concluded that not only the number of coefficients matters, the selectivity property is also important.

In [11], the Johnston filter with 32 coefficients was introduced and offered good results when used for harmonic measurement up to the 15th harmonic. For higher frequency harmonic analysis a significant improvement can be obtained if another filter from Johnston's family with 64 coefficients (J64E) is used. Due to the efficient polyphase implementation of the Johnston filters, an increase of the filter length does not have a significant influence on the computational cost compared with the v24 filters.

The performance of the filters can also be analyzed when a 1-p.u. single tone at the odd harmonic frequencies (1st to 63rd) is set to be the input signal. In Tables 2 and 3, rms values calculated for each frequency band obtained using different filters after five levels of decomposition with an input signal of a 1-p.u. single tone at the odd harmonic frequencies (25th to 39th harmonic) are given. Pointing to the middle-range frequencies $(1550 \mathrm{~Hz}$ to $1750 \mathrm{~Hz}$ ) in Table 2 one can notice that overlapping in these bands is significant and also the spectral leakage, so the error increases to $25 \%$. In Table 3, a Johnston filter with 64 coefficients is added to the analysis in order to compare its performance. It is noticeable that the errors in previously mentioned bands are significantly reduced.

Table 2. RMS values of the 13th to 20th output band of the decomposition in 5 levels using db20 and v24, when 1-p.u. single tone at the odd harmonic frequencies is set to be the input signal.

\begin{tabular}{|c|c|c|c|c|c|c|c|c|c|}
\hline \multicolumn{10}{|c|}{ Output bands } \\
\hline $\begin{array}{l}\text { Input } \\
\text { signal }\end{array}$ & filter & $\begin{array}{c}1.2-1.3 \mathrm{kHz} \\
\text { (d13) }\end{array}$ & $\begin{array}{c}1.3-1.4 \mathrm{kHz} \\
\text { (d14) }\end{array}$ & $\begin{array}{c}\text { 1.4-1.5kHz } \\
\text { (d15) }\end{array}$ & $\begin{array}{l}1.5-1.6 \mathrm{kHz} \\
\text { (d16) }\end{array}$ & $\begin{array}{c}1.6-1.7 \mathrm{kHz} \\
\text { (d17) }\end{array}$ & $\begin{array}{c}1.7-1.8 \mathrm{kHz} \\
\text { (d18) }\end{array}$ & $\begin{array}{c}\text { 1.8-1.9kHz } \\
\text { (d19) }\end{array}$ & $\begin{array}{c}1.9- \\
2 \mathrm{kHz} \\
\text { (d20) }\end{array}$ \\
\hline \multirow{2}{*}{$\begin{array}{c}1250 \\
\mathrm{~Hz}\end{array}$} & $\mathrm{db} 20$ & 0,870 & 0,009 & 0,000 & 0,0000 & 0,000 & 0,000 & 0,002 & 0,2249 \\
\hline & v24 & 0,938 & 0,000 & 0,000 & 0,000 & 0,000 & 0,000 & 0,000 & 0,108 \\
\hline \multirow{2}{*}{$\begin{array}{c}1350 \\
\mathrm{~Hz}\end{array}$} & $\mathrm{db} 20$ & 0,0098 & 0,9083 & 0,1839 & 0,0020 & 0,0008 & 0,0736 & 0,3633 & 0,0039 \\
\hline & v24 & 0,000 & 0,967 & 0,068 & 0,000 & 0,000 & 0,017 & 0,244 & 0,000 \\
\hline \multirow{2}{*}{$\begin{array}{c}1450 \\
\mathrm{~Hz}\end{array}$} & $\mathrm{db} 20$ & 0,0018 & 0,1710 & 0,8446 & 0,0091 & 0,0054 & 0,4972 & 0,1007 & 0,0011 \\
\hline & v24 & 0,000 & 0,064 & 0,904 & 0,000 & 0,000 & 0,421 & 0,030 & 0,000 \\
\hline \multirow{2}{*}{$\begin{array}{c}1550 \\
\mathrm{~Hz}\end{array}$} & $\mathrm{db} 20$ & 0,0000 & 0,0000 & 0,0083 & 0,7654 & 0,6435 & 0,0070 & 0,0000 & 0,0000 \\
\hline & v24 & 0,000 & 0,000 & 0,000 & 0,787 & 0,617 & 0,000 & 0,000 & 0,000 \\
\hline \multirow{2}{*}{$\begin{array}{c}1650 \\
\mathrm{~Hz}\end{array}$} & $\mathrm{db} 20$ & 0,0000 & 0,0000 & 0,0070 & 0,6435 & 0,7654 & 0,0083 & 0,0000 & 0,0000 \\
\hline & v24 & 0,000 & 0,000 & 0,000 & 0,617 & 0,787 & 0,000 & 0,000 & 0,000 \\
\hline \multirow{2}{*}{$\begin{array}{c}1750 \\
\mathrm{~Hz}\end{array}$} & $\mathrm{db} 20$ & 0,0011 & 0,1007 & 0,4972 & 0,0054 & 0,0091 & 0,8446 & 0,1710 & 0,0018 \\
\hline & v24 & 0,000 & 0,030 & 0,421 & 0,000 & 0,000 & 0,904 & 0,064 & 0,000 \\
\hline \multirow{2}{*}{$\begin{array}{c}1850 \\
\mathrm{~Hz}\end{array}$} & $\mathrm{db} 20$ & 0,0039 & 0,3633 & 0,0736 & 0,0008 & 0,0020 & 0,1839 & 0,9083 & 0,0098 \\
\hline & v24 & 0,000 & 0,244 & 0,017 & 0,000 & 0,000 & 0,068 & 0,967 & 0,000 \\
\hline \multirow{2}{*}{$\begin{array}{c}1950 \\
\mathrm{~Hz}\end{array}$} & $\mathrm{db} 20$ & 0,2249 & 0,0024 & 0,0000 & 0,0000 & 0,0000 & 0,0000 & 0,0094 & 0,8702 \\
\hline & v24 & 0,108 & 0,000 & 0,000 & 0,000 & 0,000 & 0,000 & 0,000 & 0,938 \\
\hline
\end{tabular}

Table 3. RMS values of the 13th to 20th output band of the decomposition in 5 levels using Johnston filters (J32D and J64E), when 1-p.u. single tone at the odd harmonic frequencies is set to be the input signal.

\begin{tabular}{|c|c|c|c|c|c|c|c|c|c|}
\hline \multicolumn{10}{|c|}{ Output bands } \\
\hline $\begin{array}{l}\text { Input } \\
\text { signal }\end{array}$ & filter & $\begin{array}{c}1.2-1.3 \mathrm{kHz} \\
\text { (d13) }\end{array}$ & $\begin{array}{c}1.3-1.4 \mathrm{kHz} \\
\text { (d14) }\end{array}$ & $\begin{array}{c}1.4-1.5 \mathrm{kHz} \\
\text { (d15) }\end{array}$ & $\begin{array}{c}1.5-1.6 \mathrm{kHz} \\
\text { (d16) }\end{array}$ & $\begin{array}{c}1.6-1.7 \mathrm{kHz} \\
\text { (d17) }\end{array}$ & $\begin{array}{c}1.7-1.8 \mathrm{kHz} \\
\text { (d18) }\end{array}$ & $\begin{array}{c}\text { 1.8-1.9kHz } \\
\text { (d19) }\end{array}$ & $\begin{array}{c}1.9- \\
2 \mathrm{kHz} \\
\text { (d20) }\end{array}$ \\
\hline \multirow{2}{*}{$\begin{array}{c}1250 \\
\mathrm{~Hz}\end{array}$} & $\mathrm{~J} 32 \mathrm{D}$ & 0,9912 & 0,0018 & 0,0000 & 0,0005 & 0,0000 & 0,0000 & 0,0000 & 0,0140 \\
\hline & J64E & 0,9972 & 0,0004 & 0,0000 & 0,0002 & 0,0000 & 0,0000 & 0,0000 & 0,0048 \\
\hline \multirow{2}{*}{$\begin{array}{c}1350 \\
\mathrm{~Hz} \\
\end{array}$} & $\mathrm{~J} 32 \mathrm{D}$ & 0,0018 & 0,9990 & 0,0106 & 0,0000 & 0,0000 & 0,0005 & 0,0510 & 0,0001 \\
\hline & J64E & 0,0004 & 1,0004 & 0,0016 & 0,0000 & 0,0000 & 0,0000 & 0,0063 & 0,0000 \\
\hline \multirow{2}{*}{$\begin{array}{c}1450 \\
\mathrm{~Hz} \\
\end{array}$} & $\mathrm{~J} 32 \mathrm{D}$ & 0,0000 & 0,0102 & 0,9636 & 0,0018 & 0,0005 & 0,2602 & 0,0028 & 0,0000 \\
\hline & J64E & 0,0000 & 0,0016 & 0,9993 & 0,0004 & 0,0000 & 0,0070 & 0,0000 & 0,0000 \\
\hline \multirow{2}{*}{$\begin{array}{c}1550 \\
\mathrm{~Hz} \\
\end{array}$} & $\mathrm{~J} 32 \mathrm{D}$ & 0,0004 & 0,0000 & 0,0015 & 0,8261 & 0,5629 & 0,0010 & 0,0000 & 0,0003 \\
\hline & J64E & 0,0002 & 0,0000 & 0,0003 & 0,9122 & 0,4103 & 0,0001 & 0,0000 & 0,0001 \\
\hline \multirow{2}{*}{$\begin{array}{c}1650 \\
\mathrm{~Hz} \\
\end{array}$} & $\mathrm{~J} 32 \mathrm{D}$ & 0,0003 & 0,0000 & 0,0010 & 0,5629 & 0,8261 & 0,0015 & 0,0000 & 0,0004 \\
\hline & J64E & 0,0001 & 0,0000 & 0,0001 & 0,4103 & 0,9122 & 0,0003 & 0,0000 & 0,0002 \\
\hline \multirow{2}{*}{$\begin{array}{c}1750 \\
\mathrm{~Hz}\end{array}$} & $\mathrm{~J} 32 \mathrm{D}$ & 0,0000 & 0,0028 & 0,2602 & 0,0005 & 0,0018 & 0,9636 & 0,0102 & 0,0000 \\
\hline & J64E & 0,0000 & 0,0000 & 0,0070 & 0,0000 & 0,0004 & 0,9993 & 0,0016 & 0,0000 \\
\hline \multirow{2}{*}{$\begin{array}{c}1850 \\
\mathrm{~Hz} \\
\end{array}$} & $\mathrm{~J} 32 \mathrm{D}$ & 0,0001 & 0,0510 & 0,0005 & 0,0000 & 0,0000 & 0,0106 & 0,9990 & 0,0018 \\
\hline & J64E & 0,0000 & 0,0063 & 0,0000 & 0,0000 & 0,0000 & 0,0016 & 1,0004 & 0,0004 \\
\hline \multirow{2}{*}{$\begin{array}{c}1950 \\
\mathrm{~Hz}\end{array}$} & J32D & 0,0140 & 0,0000 & 0,0000 & 0,0000 & 0,0005 & 0,0000 & 0,0018 & 0,9912 \\
\hline & J64E & 0,0048 & 0,0000 & 0,0000 & 0,0000 & 0,0002 & 0,0000 & 0,0004 & 0,9972 \\
\hline
\end{tabular}


Frequency characteristics of the output bands obtained after three levels of decomposition with two of the compared filters (J64E and v24) are given at Fig. 5. It can be noticed that the J64E filter has a better selectivity characteristics and lowered spectral leakage than the v24 filter. It is especially evident in the middle-frequency ranges. Frequency characteristics of the output bands obtained after five levels of decomposition results with significant difference.

\subsection{Classification of the $P Q$ disturbances}

Seven different classes are considered, including the case with no power disturbances: normal, swell, sag, harmonic, outage, sag with harmonic and swell with harmonic denoted with C1, C2, C3, C4, C5, C6, C7 (Table 4). The sampling frequency is 256 samples/cycle and the duration of the signal is ten periods. The normal frequency is assumed to be $50 \mathrm{~Hz}$. 200 signals were generated from every class with random parameters for the signal models. A test set was also generated in the same way. As described, choice of the wavelet affects the way the signal is decomposed, which for some wavelets leads to drastic changes of the accuracy of the classification algorithms. We use wavelets with different support sizes and from the usually used families: Daubechies, Coiflets, etc. Additionally, we use the Johnston filters designed for a NPR filter bank structure.

Classification results are given in Table 5. The presented results show that all tested filters provide approximately the same results. Additionally, the presented results show that for the implemented classification method the use of the Johnston filters leads to increased accuracy compared with the commonly used db4 wavelet.

Table 4. Power quality disturbance models.

\begin{tabular}{|l|l|l|l|}
\hline disturbance & class & Model & Parameters \\
\hline normal & $\mathrm{C} 1$ & $x(t)=\sin (\omega t)$ & $/$ \\
\hline swell & $\mathrm{C} 2$ & $x(t)=A\left(1+\alpha\left(u\left(t-t_{1}\right)-u\left(t-t_{2}\right)\right)\right) \sin (\omega t)$ & $0.1 \leq \alpha \leq 0.8, T \leq t_{2}-t_{1} \leq 9 T$, \\
\hline sag & $\mathrm{C} 3$ & $x(t)=A\left(1-\alpha\left(u\left(t-t_{1}\right)-u\left(t-t_{2}\right)\right)\right) \sin (\omega t)$ & $0.1 \leq \alpha \leq 0.9, T \leq t_{2}-t_{1} \leq 9 T$ \\
\hline harmonic & $\mathrm{C} 4$ & $x(t)=A\left(\alpha_{1} \sin (\omega t)+\alpha_{3} \sin (3 \omega t)+\alpha_{5} \sin (5 \omega t)+\alpha_{7} \sin (7 \omega t)\right)$ & $\begin{array}{l}0.05 \leq \alpha_{3} \leq 0.15,0.05 \leq \alpha_{5} \leq 0.15,0.05 \leq \alpha_{7} \leq 0.15, \\
\sum \alpha_{i}^{2}=1\end{array}$ \\
\hline $\begin{array}{l}\text { outage with } \\
\text { sag }\end{array}$ & $\mathrm{C} 5$ & $\begin{array}{l}x(t)=A\left(1-\alpha\left(u\left(t-t_{1}\right)-u\left(t-t_{2}\right)\right)\right) \sin (\omega t) \\
\left(\alpha_{1} \sin (\omega t)+\alpha_{3} \sin (3 \omega t)+\alpha_{5} \sin (5 \omega t)\right)\end{array}$ & $0.9 \leq \alpha \leq 1, T \leq t_{2}-t_{1} \leq 9 T$ \\
\hline $\begin{array}{l}x \text { swell with } \\
\text { harmonic }\end{array}$ & $\mathrm{C} 7$ & $\begin{array}{l}x(t)=A\left(1+\alpha\left(u\left(t-t_{1}\right)-u\left(t-t_{2}\right)\right)\right) \\
\left(\alpha_{1} \sin (\omega t)+\alpha_{3} \sin (3 \omega t)+\alpha_{5} \sin (5 \omega t)\right)\end{array}$ & $\begin{array}{l}0.1 \leq \alpha \leq 0.9, T \leq t_{2}-t_{1} \leq 9 T \\
0.05 \leq \alpha_{3} \leq 0.15,0.05 \leq \alpha_{5} \leq 0.15, \sum \alpha_{i}^{2}=1\end{array}$ \\
\hline
\end{tabular}

Table 5. Results from the classification with the use of different wavelets.

\begin{tabular}{|c|c|c|c|c|c|c|c|c|c|c|c|c|c|c|c|}
\hline $\begin{array}{l}\frac{\pi}{0} \\
\stackrel{\pi}{J}\end{array}$ & $\begin{array}{l}\stackrel{0}{0} \\
\stackrel{0}{0} \\
\stackrel{0}{0}\end{array}$ & $\frac{\frac{N}{ \pm}}{\frac{\tilde{c}}{0}}$ & 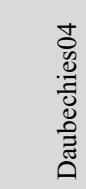 & 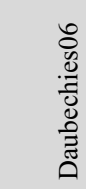 & 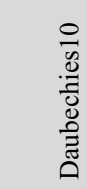 & 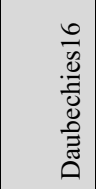 & 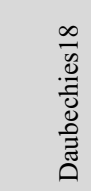 & 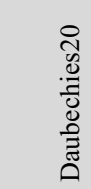 & 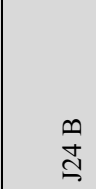 & $\begin{array}{l}\text { U } \\
\stackrel{\mathbb{J}}{J}\end{array}$ & $\begin{array}{l}\sim \\
\stackrel{\sim}{n}\end{array}$ & 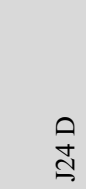 & 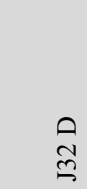 & $\stackrel{\omega}{N}$ & 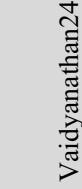 \\
\hline $\mathrm{C} 1$ & 100 & 100 & 100 & 100 & 100 & 100 & 100 & 100 & 100 & 100 & 100 & 100 & 100 & 100 & 100 \\
\hline $\mathrm{C} 2$ & 100 & 100 & 100 & 100 & 100 & 100 & 100 & 100 & 100 & 100 & 100 & 100 & 100 & 100 & 99,5 \\
\hline C3 & 90 & 89 & 78,5 & 91 & 82 & 82,5 & 80,5 & 75,5 & 81,5 & 81,5 & 79 & 80,5 & 79,5 & 78,5 & 78 \\
\hline $\mathrm{C} 4$ & 100 & 100 & 100 & 100 & 100 & 100 & 100 & 100 & 100 & 100 & 100 & 100 & 100 & 100 & 100 \\
\hline $\mathrm{C} 5$ & 91,5 & 91,5 & 80,5 & 93 & 78,5 & 79 & 88 & 83 & 84,5 & 86 & 83 & 85,5 & 84 & 84 & 78,5 \\
\hline C6 & 98 & 98 & 100 & 98,5 & 78 & 99 & 100 & 98 & 100 & 100 & 100 & 100 & 100 & 100 & 99 \\
\hline $\mathrm{C} 7$ & 100 & 100 & 100 & 100 & 93 & 100 & 100 & 100 & 100 & 100 & 100 & 100 & 100 & 100 & 100 \\
\hline overall & 97,07 & 96,93 & 94,14 & 97,50 & 90,21 & 94,36 & 95,50 & 93,79 & 95,14 & 95,36 & 94,57 & 95,14 & 94,79 & 94,64 & 93,57 \\
\hline
\end{tabular}


Furthermore, we test the accuracy of the algorithm in the presence of noise with the use of different wavelets. We add white Gaussian noise to the signals from the test set. Different levels of noise with the signal-to-noise ratio (SNR) values ranging from 20 to $50 \mathrm{~dB}$ are added. Classification results obtained with the implemented classification method in presence of noise are given in Table 6. It can be seen from the presented results that the use of Johnston filters with 32 coefficients adds robustness to the classification methods in the presence of noise. The power quality disturbances can be classified with high precision even if $20 \mathrm{~dB}$ noise is added to the disturbance signals. This is not the case with the other wavelet families, where accuracy of the classification is significantly reduced as the SNR becomes smaller.

The same experiments using the multiclass logistic regression classification method were performed in [13]. The obtained results are very similar to the results presented in this paper.

Table 6. Overall accuracy of the SVM classification method with added noise to tested signals

\begin{tabular}{|c|c|c|c|c|c|c|c|}
\hline Wavelet & $20 \mathrm{~dB}$ & $25 \mathrm{~dB}$ & $30 \mathrm{~dB}$ & $35 \mathrm{~dB}$ & $40 \mathrm{~dB}$ & $45 \mathrm{~dB}$ & $50 \mathrm{~dB}$ \\
\hline Beylkin 18 & 81,79 & 87,86 & 94,93 & 96,14 & 95,93 & 96,21 & 96,07 \\
\hline Coiflet06 & 64,71 & 83,36 & 94,36 & 96,43 & 96,86 & 97,00 & 97,07 \\
\hline Coiflet30 & 57,50 & 80,43 & 93,93 & 95,71 & 95,79 & 96,07 & 96,00 \\
\hline Daub04 & 84,79 & 85,50 & 86,14 & 94,00 & 93,93 & 94,21 & 94,21 \\
\hline Daub06 & 70,14 & 88,64 & 95,64 & 97,00 & 97,29 & 97,5 & 97,50 \\
\hline Daub10 & 49,29 & 63,36 & 79,36 & 86,64 & 89,07 & 89,64 & 90,00 \\
\hline Daub20 & 74,86 & 91,07 & 93,57 & 93,57 & 93,71 & 93,86 & 93,79 \\
\hline $\mathrm{J} 24 \mathrm{~B}$ & 86,00 & 94,36 & 94,86 & 95,5 & 95,14 & 95,14 & 95,14 \\
\hline J24 C & 85,14 & 93,57 & 95,00 & 95,07 & 95,29 & 95,21 & 95,36 \\
\hline $\mathrm{J} 32 \mathrm{C}$ & 94,36 & 94,43 & 94,5 & 94,57 & 94,64 & 94,57 & 94,57 \\
\hline J24 D & 82,43 & 93,21 & 95,14 & 95,21 & 95,00 & 95,14 & 95,14 \\
\hline J32 D & 94,07 & 94,71 & 94,79 & 94,79 & 94,71 & 94,79 & 94,79 \\
\hline $\mathrm{J} 32 \mathrm{E}$ & 93,29 & 94,64 & 94,79 & 94,71 & 94,71 & 94,57 & 94,64 \\
\hline V24 & 64,14 & 82,29 & 92,21 & 93,07 & 93,21 & 93,43 & 93,5 \\
\hline
\end{tabular}

\section{Conclusions}

In this paper we have analyzed the influence of the NPR QMF filter banks for PQ analysis: harmonics measurement and detection PQ disturbances. Frequency characteristics of the lowpass and high-pass filter pairs used in the implementation of the wavelet transform have a strong influence on the accuracy of harmonics measurement. This influence is especially evident when high frequency harmonics have to be analyzed, which require a larger level of WPT decomposition to be performed. Filters with good frequency selectivity present a lower level of spectral leakage and are suitable for measurement of high order harmonics. In that sense Johnston filters with 64 coefficients are one possible solution.

The obtained results from the simulations made, also indicated that Johnston filters are a good choice for application in the power quality classification methods, especially in the noise scenario. With the use of Johnston filters with 32 coefficients the accuracy of the classification method is not significantly reduced even if $20 \mathrm{~dB}$ noise is added to the disturbance signals.

Additionally, due to efficient polyphase implementation of the Johnston filters, an increase of the filter length does not have significant influence on the computational cost compared with the commonly used wavelets. 


\section{References}

[1] Targosz R., Manson J. (2007), Pan-European power quality survey. In Proceedings of 9th IEEE Electrical Power Quality and Utilization Conference, Barcelona, Spain, 9-11.

[2] IEEE Recommended practice for monitoring electric power quality, Std. 1159-2009

[3] Ibrahim W. R. A., Morcos M. M. (2002). Artificial intelligence and advanced mathematical tools for power quality applications: A survey. IEEE Trans. Power Delivery, 17 (2), 668-673.

[4] Szmajda M., Górecki K., Mroczka J. (2010). Gabor transform, SPWVD, Gabor-Wigner transform and wavelet transform - tools for power quality monitoring. Metrology and Measurement Systems, 17(3), 383-396.

[5] Chen S., Zhu H. Y., (2007). Wavelet transform for processing power quality disturbances, EURASIP J. Adv. Signal Process. 2007.

[6] Barros J., Diego R. (2008). Analysis of harmonics in power systems using wavelet packet transform. IEEE Trans. Instrumentation and Measurement, 57, 63-69.

[7] Hamid E., Yokoyama Kawasaki Z. (2002). Rms and power measurements: A wavelet packet transform approach. Trans. Institute of Electrical Engineers of Japan, 122-B (5), 599-606.

[8] Barros J., Diego R., Apraiz M. de. (2012) Applications of wavelet transform for analysis of harmonic distortion in power systems: A review. IEEE Trans. on Instrumentation and Measurement, 61 (10)

[9] Electromagnetic Compatibility (EMC) - Part 4-7: Testing and measurement techniques - general guide on harmonics and interharmonics measurement and instrumentation for power supply systems and equipment connected thereto, IEC 61000-4-7, 2002.

[10] Vaidyanathan P. (1993). Multirate systems and filter banks, Prentice-Hall.

[11] Taskovski D., Koleva Lj. (2012). Measurement of harmonics in power systems using near perfect reconstruction filter banks. IEEE Trans. Power Delivery, 27(2), 1025-1026,

[12] Johnston J.D. (1980). A filter family designed for use in quadrature mirror filter banks. In Proc. IEEE Inter. Conf. Acoustics, Speech, and Signal Processing, 291-294.

[13] Koleva Lj., Taskovski D., Milchevski A., Dimchev V. (2012) Application of near perfect reconstruction filter banks in power quality disturbances classification methods. In Proc. IEEE Int. Workshop on Applied Measurement for Power Systems, Aachen, Germany. CD-ROM.

[14] Santoso S., Powers E.J., Grady W.M., Hofmann P. (1996). Power quality assessment via wavelet transform analysis. IEEE Trans. Power Delivery, 11 (2). 924-930.

[15] Boser B. E., Guyon I., Vapnik V. (1992). A training algorithm for optimal margin classifers. In Proc. of the Fifth Workshop on Computational Learning Theory, 144-152.

[16] Cortes C., Vapnik, V. (1995). Support-vector network. Machine Learning, 20, 273-297.

[17] Chang C.C., Lin C.J. LIBSVM - A Library for Support Vector Machines, http://www.csie.ntu.edu.tw/ cjlin/libsvm/

[18] Bollen M., Ribeiro P., Gu I., Duque C. A. (2010) Trends, challenges and opportunities in power quality research, European Transactions on Power Quality, 20.

[19] Electromagnetic Compatibility (EMC) - Part 3-2: Limits for harmonic current emissions, 2009, IEC 61000-3-2

[20] Gherasim G., Driesen J., Belmans R. (2006). Real-time implementation and comparison of time-varying harmonic measurement methods. In Proc. IEEE Power Systems Conference \& Exposition, 239-245. 